\title{
APPROXIMATION OF QUANTUM GRAPH VERTEX COUPLINGS BY SCALED SCHRÖDINGER OPERATORS ON THIN BRANCHED MANIFOLDS
}

\author{
PAVEL EXNER AND OLAF POST
}

\begin{abstract}
We discuss approximations of vertex couplings of quantum graphs using families of thin branched manifolds. We show that if a Neumann type Laplacian on such manifolds is amended by suitable potentials, the resulting Schrödinger operators can approximate non-trivial vertex couplings. The latter include not only the $\delta$-couplings but also those with wavefunctions discontinuous at the vertex. We work out the example of the symmetric $\delta^{\prime}$-couplings and conjecture that the same method can be applied to all couplings invariant with respect to the time reversal.
\end{abstract}

\section{INTRODUCTION}

The quantum graph models represent a simple and versatile tool to study numerous physical phenomena. The current state of art in this field is described in the recent proceedings volume $\left[\mathrm{EKK}^{+} 08\right]$ to which we refer for an extensive bibliography.

One of the big questions in this area is the physical meaning of quantum graph vertex coupling. The general requirement of self-adjointness admits boundary conditions containing a number of parameters, and one would like to understand how to choose these when a quantum graph model is applied to a specific physical situation. One natural idea is to approximate the graph in question by a family of "fat graphs", i.e. tube-like manifolds built around the graph "skeleton", equipped with a suitable second-order differential operator. Such systems have no ad hoc parameters and one can try to find what boundary condition arise when the manifold is squeezed to the graph.

The question is by no means easy and the answer depends on the type of the operator chosen. If it is the Laplacian with Dirichlet boundary conditions one has to employ an energy renormalisation because the spectral threshold given by the lowest transverse eigenvalue blows up to infinity as the tube diameter tends to zero. If one chooses the reference point between the thresholds, the limiting boundary conditions are determined by the scattering on the respective "fat star" manifold [MV07]. If, on the other hand, the threshold energy is subtracted, the limit gives generically a decoupled graph, i.e. the family of edges with Dirichlet conditions at their endpoints [P05, MV07, DT06]. One can nevertheless get a non-trivial coupling in the limit if the tube network exhibits a threshold resonance G08, ACF07, and moreover, using a more involved limiting process one can get also boundary conditions with richer spectral properties [CE07].

The case when the fat graph supports a Laplacian of Neumann type is better understood and the limit of all types of spectra as well as of resonances has been worked out [FW93, RS01, KuZ01, EP05, EP07, G08, EP08,. Moreover, convergence of resolvents etc. has been shown in [Sai00, P06, EP07]. Of course, no energy renormalisation is needed in this case. On the other hand, the limit yields only the simplest boundary conditions called free or Kirchhoff.

The aim of this paper is to show that one can do better in the Neumann case if the Laplacian is replaced by suitable families of Schrödinger operators with properly scaled potentials. Such approximations have been shown to work on graphs themselves [E97,

Date: June 6, 2018 File: pot-approx5.tex. 
ENZ01, here we are going to "lift" them to the tube-like manifolds. 1 . First we will show that using potentials supported by the vertex regions of the manifold with the "natural" scaling, as $\varepsilon^{-1}$ where $\varepsilon$ is the tube radius parameter, we can get the so-called $\delta$-coupling, the one-parameter family with the wavefunctions continuous everywhere, including at the vertex. This shows, in particular, that one cannot achieve such an approximation in a purely geometric way, with a curvature-induced potential of the type [DEK01], because the latter scales typically as $\varepsilon^{-2}$. As main result in this case, we show the convergence of the spectra and the resolvents (cf. Theorems 3.3 3.7).

On the other hand, the $\delta$-coupling is only a small part in the set of all admissible couplings; in a vertex joining $n$ edges the boundary conditions contain $n^{2}$ parameters. Here we use the seminal idea of Cheon and Shigehara [CS98] applied to the graph case in CE04 and generalised in [ET06, ET07]. For simplicity we will work out in this paper the example of the so-called symmetric $\delta^{\prime}$-coupling, in short $\delta_{\mathrm{s}}^{\prime}$, a one-parameter family which is a counterpart of $\delta$, by using the result of [CE04] and "lifting" it to the manifold. We show that such a coupling is approximated with a potential in the vertex region together with potentials at the edges with compact supports approaching the vertex, all properly scaled, cf. Theorem 4.7. The speed with which the potentials are "coming together" must be slower than the squeezing; the rate between the two we obtain is surely not optimal.

We have no doubts that in the same way one can lift to the manifolds the more general limiting procedure devised in [ET07 which gives rise to a $\left(\begin{array}{c}n+1 \\ 2\end{array}\right)$-parameter family of boundary conditions, namely those which are invariant with respect to the time reversal. Such an extension would be technically demanding, however, and in order not to burden this paper with a complicated notation and voluminous estimations we postpone it to a later work.

Let us survey the contents of the paper. In the next section we define the graph and manifold models and provide necessary estimates. In Section 3 we prove the convergence in the $\delta$-coupling case. For simplicity we restrict ourselves to a star-shaped graph with a single vertex; the approximation bears a local character and extends easily to more complex graphs. Finally in Section 4 we extend the result to the $\delta_{\mathrm{s}}^{\prime}$-coupling case and comment on the applicability of the method to more general couplings.

\section{THE GRAPH AND MANIFOLD MODELS}

2.1. Graph model. Let us start with a simple example of a star-shaped metric graph $G=I_{v}$ having only one vertex $v$ and $\operatorname{deg} v$ adjacent edges $E=E_{v}$ of length $\ell_{e} \in(0, \infty]$, so we can think of $E=\{1, \ldots, \operatorname{deg} v\}$. We identify the (metric) edge $e$ with the interval $I_{e}:=\left(0, \ell_{e}\right)$ oriented in such a way that 0 corresponds to the vertex $v$. Moreover, the metric graph $G=I_{v}$ is given by the abstract space $I_{v}:=\cup_{e} \bar{I}_{e} / \sim$ where $\bullet$ denotes the disjoint union, and where the equivalence relation $\sim$ identifies the points $0 \in \bar{I}_{e}$ with the vertex $v$. The basic Hilbert space is $\mathrm{L}_{2}(G):=\bigoplus_{e \in E} \mathrm{~L}_{2}\left(I_{e}\right)$ with norm given by

$$
\|f\|^{2}=\|f\|_{G}^{2}=\sum_{e \in E} \int_{0}^{\ell_{e}}|f(s)|^{2} \mathrm{~d} s .
$$

The decoupled Sobolev space of order $k$ is defined as

$$
\mathrm{H}_{\max }^{k}(G):=\bigoplus_{e \in E} \mathrm{H}^{k}\left(I_{e}\right)
$$

\footnotetext{
${ }^{1}$ Another approach to approximation of nontrivial vertex conditions was proposed recently in $\mathrm{Pa} 07 \mathrm{~b}$, $\mathrm{Pa} 07 \mathrm{a}$
} 
together with its natural norm. Let $\underline{p}=\left\{p_{e}\right\}_{e}$ be a vector consisting of the weights $p_{e}>0$ for $e \in E$. The Sobolev space associated to the weight $\underline{p}$ is given by

$$
\mathrm{H}_{\underline{p}}^{1}(G):=\left\{f \in \mathrm{H}_{\max }^{1}(G) \mid \underline{f} \in \mathbb{C} \underline{p}\right\},
$$

where $\underline{f}:=\left\{f_{e}(0)\right\}_{e} \in \mathbb{C}^{\operatorname{deg} v}$ is the evaluation vector of $f$ at the vertex $v$ and $\mathbb{C} p$ is the complex span of $\underline{p}$. We use the notation

$$
\underline{f}=f(v) \underline{p}, \quad \text { i.e., } \quad f_{e}=f(v) p_{e}
$$

for all $e \in E$. In particular, if $\underline{p}=(1, \ldots, 1)$, we arrive at the continuous Sobolev space $\mathrm{H}^{1}(G):=\mathrm{H}_{\underline{p}}^{1}(G)$. The standard Sobolev trace estimate

$$
|g(0)|^{2} \leq a\left\|g^{\prime}\right\|_{(0, \ell)}^{2}+\frac{2}{a}\|g\|_{(0, \ell)}^{2}
$$

for $g \in \mathrm{H}^{1}(0, \ell)$ and $0<a \leq \ell$ ensures that $\mathrm{H}_{\underline{p}}^{1}(G)$ is a closed subspace of $\mathrm{H}_{\max }^{1}(G)$, and therefore itself a Hilbert space. A simple consequence is the following claim.

Lemma 2.1. We have

$$
|f(v)|^{2} \leq|\underline{p}|^{-2}\left(a\left\|f^{\prime}\right\|_{G}^{2}+\frac{2}{a}\|f\|_{G}^{2}\right)
$$

for $f \in \mathrm{H}_{\underline{p}}^{1}(G)$ and $0<a \leq \ell_{-}:=\min _{e \in E} \ell_{e}$, the minimal length at the vertex $v$.

We define various Laplacians on the metric graph via their quadratic forms. Let us start with the (weighted) free Laplacian $\Delta_{G}$ defined via the quadratic form $\mathfrak{d}=\mathfrak{d}_{G}$ given by

$$
\mathfrak{d}(f):=\left\|f^{\prime}\right\|_{G}^{2}=\sum_{e}\left\|f_{e}^{\prime}\right\|_{I_{e}}^{2} \quad \text { and } \quad \operatorname{dom} \mathfrak{d}:=\mathrm{H}_{\underline{p}}^{1}(G)
$$

for a fixed $\underline{p}$ (the forms and the corresponding operators should be labelled by the weight $\underline{p}$, of course, but we drop the index, in particular, because we are most interested in the case $p=(1, \ldots, 1))$. Note that $\mathfrak{d}$ is a closed form since the norm associated to the quadratic form $\mathfrak{d}$ is precisely the Sobolev norm given by $\|f\|_{\mathrm{H}^{1}(G)}^{2}=\left\|f^{\prime}\right\|_{G}^{2}+\|f\|_{G}^{2}$.

The Laplacian with $\delta$-coupling of strength $q$ is defined via the quadratic form $\mathfrak{h}=\mathfrak{h}_{(G, q)}$ given by

$$
\mathfrak{h}(f):=\left\|f^{\prime}\right\|_{G}^{2}+q(v)|f(v)|^{2} \quad \text { and } \quad \operatorname{dom} \mathfrak{h}:=\mathrm{H}_{\underline{p}}^{1}(G) .
$$

The $\delta$-coupling is a "small" perturbation of the free Laplacian, namely we have:

Lemma 2.2. The form $\mathfrak{h}_{(G, q)}$ is relatively form-bounded with respect to the free form $\mathfrak{d}_{G}$ with relative bound zero, i.e., for any $\eta>0$ there exists $C_{\eta}>0$ such that

$$
|\mathfrak{h}(f)-\mathfrak{d}(f)|=|q(v)||f(v)|^{2} \leq \eta \mathfrak{d}(f)+C_{\eta}\|f\|_{G}^{2} .
$$

Proof. It is again a simple consequence of Lemma 2.1. Since we need the precise behaviour of the constant $C_{\eta}$, we give a short proof here. From Lemma 2.1 we conclude that

$$
|\mathfrak{h}(f)-\mathfrak{d}(f)| \leq|q(v)||\underline{p}|^{-2}\left(a \mathfrak{d}(f)+\frac{2}{a}\|f\|_{G}^{2}\right) .
$$

for any $0<a \leq \ell_{-}$. Set $a:=\min \left\{\eta|\underline{p}|^{2} /|q(v)|, \ell_{-}\right\}$and

$$
C_{\eta}:=2 \max \left\{\frac{|q(v)|^{2}}{\eta|\underline{p}|^{4}}, \frac{|q(v)|}{\ell_{-}|\underline{p}|^{2}}\right\},
$$

then the desired estimate follows.

One can see that the norms associated to $\mathfrak{h}$ and $\mathfrak{d}$ are equivalent and, in particular, setting $\eta=1 / 2$ in the above estimate yields we get: 
Corollary 2.3. The quadratic form $\mathfrak{h}$ is closed and obeys the estimate

$$
\mathfrak{d}(f) \leq 2\left(\mathfrak{h}(f)+C_{1 / 2}\|f\|_{G}^{2}\right) .
$$

The operator $H=H_{(G, q)}$ associated to $\mathfrak{h}$ acts as $(H f)_{e}=-f_{e}^{\prime \prime}$ on each edge and satisfies the conditions

$$
\frac{f_{e_{1}}(0)}{p_{e_{1}}}=\frac{f_{e_{2}}(0)}{p_{e_{2}}}=: f(v) \quad \text { and } \quad \sum_{e} p_{e} f_{e}^{\prime}(0)=q(v) f(v)
$$

for any pair $\left(e_{1}, e_{2}\right)$ of edges meeting at the vertex $v$. We use the formal notation

$$
H=H_{(G, q)}=\Delta_{G}+q(v) \delta_{v} ;
$$

note that $\Delta_{G}$ is a non-negative operator by definition. In order to compare the "free" quadratic form with the graph norm of $H$ we need the following estimate:

Lemma 2.4. We have

$$
\|f\|_{\mathrm{H}^{1}(G)}^{2}=\mathfrak{d}(f)+\|f\|_{G}^{2} \leq 2 \max \left\{C_{1 / 2}, \sqrt{2}\right\}\|(H-\mathrm{i}) f\|_{G}^{2}
$$

for $f \in \operatorname{dom} H \subset \operatorname{dom} \mathfrak{h}=\mathrm{H}_{\underline{p}}^{1}(G)$.

Proof. Using the estimate of Corollary 2.3, we obtain

$$
\mathfrak{d}(f)+\|f\|^{2} \leq 2\left(\mathfrak{h}(f)+\left(C_{1 / 2}+1\right)\|f\|^{2}\right) \leq 2\left|\mathfrak{h}(f)+\|f\|^{2}\right|+2 C_{1 / 2}\|f\|^{2} .
$$

Moreover, the first term can be estimated as

$$
\begin{array}{r}
\left|\mathfrak{h}(f)+\|f\|^{2}\right|^{2} \leq 2\left(\mathfrak{h}(f)^{2}+\|f\|^{4}\right)=2\left|\mathfrak{h}(f)-\mathrm{i}\|f\|^{2}\right|^{2}=2|\langle f,(H-\mathrm{i}) f\rangle|^{2} \\
\leq 2\|f\|^{2}\|(H-\mathrm{i}) f\|^{2} .
\end{array}
$$

Finally, we apply the estimate $\|f\| \leq\|(H-\mathrm{i}) f\|$ to obtain the result.

Remark 2.5. Note that we have not said anything about the boundary conditions at the free ends of the edges of finite length if there are any. As we employ the Sobolev space $\mathrm{H}_{p}^{1}(G)$ for the domain, we implicitly introduce Neumann conditions for the operator, $f_{e}^{\prime}\left(\ell_{e}\right)=0$. However, one can choose any other condition at the free ends, or to construct more complicated graphs by putting the star graphs together.

2.2. Manifold model of the "fat" graph. Let us now define the other element of the approximation we are going to construct. For a given $\varepsilon \in\left(0, \varepsilon_{0}\right]$ we associate a $d$-dimensional manifold $X_{\varepsilon}$ to the graph $G$ in the following way. To the edge $e \in E$ and the vertex $v$ we ascribe the Riemannian manifolds

$$
X_{\varepsilon, e}:=I_{e} \times \varepsilon Y_{e} \quad \text { and } \quad X_{\varepsilon, v}:=\varepsilon X_{v},
$$

respectively, where $\varepsilon Y_{e}$ is a manifold $Y_{e}$ equipped with metric $h_{\varepsilon, e}:=\varepsilon^{2} h_{e}$ and $\varepsilon X_{\varepsilon, v}$ carries the metric $g_{\varepsilon, v}=\varepsilon^{2} g_{v}$; here $h_{e}$ and $g_{v}$ are $\varepsilon$-independent metrics on $Y_{e}$ and $X_{v}$, respectively. We identify the boundary component $\partial_{e} X_{\varepsilon, v}=\varepsilon \partial_{e} X_{v}$ of $\partial X_{\varepsilon, v}=\varepsilon \partial X_{v}$ with $\partial_{v} X_{\varepsilon, e}=\varepsilon \partial_{v} X_{e}=\{0\} \times \varepsilon Y_{e}$ and call the resulting manifold $X_{\varepsilon}$. We refer to the unscaled manifold (which conventionally means $\varepsilon=1$ ) as to $X$. In particular, the manifold $X$ consists of the number $\operatorname{deg} v$ of straight cylinders with cross-section $Y_{e}$ and a vertex neighbourhood manifold $X_{v}$ containing the boundary components $Y:=\cup_{e} Y_{e}$. Without loss of generality, we may assume that each cross-section $Y_{e}$ is connected, otherwise we replace the edge $e$ by as many edges as is the number of connected components. We denote the boundary component of $\partial X_{v}$ at the edge $e$ by $\partial_{e} X_{v}$ and the boundary component of $\partial X_{e}$ at the vertex $v$ by $\partial_{v} X_{e}=\{0\} \times Y_{e}$. Note that these two boundaries are identified in the entire manifold $X$. Similarly, we denote by $\partial_{e} X_{\varepsilon, v}=\varepsilon \partial_{e} X_{v}$ and $\partial_{v} X_{\varepsilon, e}=$

\footnotetext{
${ }^{2}$ The straightness here refers to the intrinsic geometry only. We do not assume in general that the manifolds $X_{\varepsilon}$ are embedded, for instance, into a Euclidean space, see also Remark 2.6.
} 
$\varepsilon \partial_{v} X_{e}$ their scaled versions. For convenience, we will always use the $\varepsilon$-independent coordinates $(s, y) \in X_{e}=I_{e} \times Y_{e}$ and $x \in X_{v}$, so that the radius-type parameter $\varepsilon$ only enters via the Riemannian metrics.

The entire manifold $X_{\varepsilon}$ may or may not have a boundary $\partial X_{\varepsilon}$, depending on whether there is at least one finite edge length $\ell_{e}<\infty$ or one "transverse" manifold $Y_{e}$ has a non-empty boundary. In such a situation, we assume that $X_{\varepsilon}$ is open in $\bar{X}_{\varepsilon}=X_{\varepsilon} \bullet \partial X_{\varepsilon}$. A particular case is represented by embedded manifolds which deserve a comment:

Remark 2.6. Note that the above setting contains the case of the $\varepsilon$-neighbourhood of an embedded graph $G \subset \mathbb{R}^{2}$, but only up to a longitudinal error of order of $\varepsilon$. The manifold $X_{\varepsilon}$ itself does not form an $\varepsilon$-neighbourhood of a metric graph embedded in some ambient space, since the vertex neighbourhoods cannot be fixed in the ambient space unless one allows slightly shortened edge neighbourhoods. Nevertheless, introducing $\varepsilon$-independent coordinates also in the longitudinal direction simplifies the comparison of the Laplacian on the metric graph and the manifold, and the error made is of order of $O(\varepsilon)$, as we will see in Lemma 2.7 for a single edge.

The basic Hilbert space of the manifold model is

$$
\mathrm{L}_{2}\left(X_{\varepsilon}\right)=\bigoplus_{e}\left(\mathrm{~L}_{2}\left(I_{e}\right) \otimes \mathrm{L}_{2}\left(\varepsilon Y_{e}\right)\right) \oplus \mathrm{L}_{2}\left(\varepsilon X_{v}\right)
$$

with the norm given by

$$
\|u\|_{X_{\varepsilon}}^{2}=\sum_{e \in E} \varepsilon^{d-1} \int_{X_{e}}|u|^{2} \mathrm{~d} y_{e} \mathrm{~d} s+\varepsilon^{d} \int_{X_{v}}|u|^{2} \mathrm{~d} x_{v}
$$

where $\mathrm{d} x_{e}=\mathrm{d} y_{e} \mathrm{~d} s$ and $\mathrm{d} x_{v}$ denote the Riemannian volume measures associated to the (unscaled) manifolds $X_{e}=I_{e} \times Y_{e}$ and $X_{v}$, respectively. In the last formula we have employed the appropriate scaling behaviour, $\mathrm{d} x_{\varepsilon, e}=\varepsilon^{d-1} \mathrm{~d} y_{e} \mathrm{~d} s$ and $\mathrm{d} x_{\varepsilon, v}=\varepsilon^{d} \mathrm{~d} x_{v}$.

Denote by $\mathrm{H}^{1}\left(X_{\varepsilon}\right)$ the Sobolev space of order one, the completion of the space of smooth functions with compact support under the norm given by $\|u\|_{\mathrm{H}^{1}\left(X_{\varepsilon}\right)}^{2}=\|\mathrm{d} u\|_{X_{\varepsilon}}^{2}+$ $\|u\|_{X_{\varepsilon}}^{2}$. As in the case of the metric graphs, we define the Laplacian $\Delta_{X_{\varepsilon}}$ on $X_{\varepsilon}$ via its quadratic form

$$
\mathfrak{d}_{\varepsilon}(u):=\|\mathrm{d} u\|_{X_{\varepsilon}}^{2}=\sum_{e \in E} \varepsilon^{d-1} \int_{X_{e}}\left(\left|u^{\prime}(s, y)\right|^{2}+\frac{1}{\varepsilon^{2}}\left|\mathrm{~d}_{Y_{e}} u\right|_{h_{e}}^{2}\right) \mathrm{d} y_{e} \mathrm{~d} s+\varepsilon^{d-2} \int_{X_{v}}|\mathrm{~d} u|_{g_{v}}^{2} \mathrm{~d} x_{v}
$$

where $u^{\prime}$ denotes the longitudinal derivative, $u^{\prime}=\partial_{s} u$, and $\mathrm{d} u$ is the exterior derivative of $u$. As before, the form $\mathfrak{d}_{\varepsilon}$ is closed by definition. Adding a potential, we define the Hamiltonian $H_{\varepsilon}$ as the operator associated with the form $\mathfrak{h}_{\varepsilon}=\mathfrak{h}_{\left(X_{\varepsilon}, Q_{\varepsilon}\right)}$ given by

$$
\mathfrak{h}_{\varepsilon}=\|\mathrm{d} u\|_{X_{\varepsilon}}^{2}+\left\langle u, Q_{\varepsilon} u\right\rangle_{X_{\varepsilon}}
$$

where the potential $Q_{\varepsilon}$ has support only in the (unscaled) vertex neighbourhood $X_{v}$ and

$$
Q_{\varepsilon}(x)=\frac{1}{\varepsilon} Q(x)
$$

where $Q=Q_{1}$ is a fixed bounded and measurable function on $X_{v}$. The reason for this particular scaling will become clear in the proof of Lemma 3.2. Roughly speaking, it comes from the fact that vol $X_{\varepsilon, v}$ is of order $\varepsilon^{d}$, whereas the $(d-1)$-dimensional transverse volume $\operatorname{vol} Y_{\varepsilon, e}$ is of order $\varepsilon^{d-1}$. The operators $H_{\varepsilon}$ and $\Delta_{\varepsilon}$ are associated to forms $\mathfrak{h}_{\varepsilon}$ and $\mathfrak{d}_{\varepsilon}$, respectively; note that $\Delta_{\varepsilon}=\Delta_{X_{\varepsilon}} \geq 0$ is the usual (Neumann) Laplacian on $X_{\varepsilon}$. As usual the Neumann boundary condition occurs only in the operator domain if $\partial X_{\varepsilon} \neq \emptyset$. We postpone for a moment the check that $H_{\varepsilon}$ is relatively form-bounded with respect to $\Delta_{X_{\varepsilon}}$, see Lemma 2.10 below. 
Let us compare the two cylindrical neighbourhoods, $X_{\varepsilon, e}=I \times \varepsilon Y_{e}$ and $\widetilde{X}_{\varepsilon, e}=I_{\varepsilon} \times \varepsilon Y_{e}$, on edges of length $\ell>0$ and $\ell_{\varepsilon}=(1-\varepsilon) \ell$, respectively. The result for the entire space $X_{\varepsilon}$ then follows by combining the estimates on the edges and the fact that the potential is only supported on the vertex neighbourhoods. The verification of the conditions of $\delta$-closeness in the next lemma is straightforward; for more details on $\delta$-closeness we refer to [P06, App. A] or [P08].

Lemma 2.7. Let $\mathscr{H}_{e}:=\mathrm{L}_{2}\left(X_{\varepsilon, e}\right)$ and $\widetilde{\mathscr{H}_{e}}:=\mathrm{L}_{2}\left(\widetilde{X}_{\varepsilon, e}\right)$. Moreover, define

$$
\begin{array}{ll}
J_{e}: \mathscr{H}_{e} \longrightarrow \widetilde{\mathscr{H}}_{e} & \left(J_{e} f\right)(\widetilde{s}, y):=f\left((1-\varepsilon)^{-1} \widetilde{s}, y\right), \\
J_{e}^{\prime}: \widetilde{\mathscr{H}_{e}} \longrightarrow \mathscr{H}_{e} & \left(J_{e}^{\prime} u\right)(s, y):=f((1-\varepsilon) s, y) .
\end{array}
$$

Then the quadratic forms $\mathfrak{d}_{\varepsilon}(f):=\|f\|_{X_{\varepsilon, e}}^{2}$ and $\widetilde{\mathfrak{d}}_{\varepsilon}(u):=\|u\|_{\widetilde{X}_{\varepsilon, e}}^{2}$ with $\operatorname{dom} \mathfrak{d}_{\varepsilon}=\mathrm{H}^{1}\left(X_{\varepsilon, e}\right)$ and $\operatorname{dom} \widetilde{\mathfrak{d}}_{\varepsilon}=\mathrm{H}^{1}\left(\widetilde{X}_{\varepsilon, e}\right)$ are $\delta_{\varepsilon}$-close with $\delta_{\varepsilon}=2 \varepsilon /(1-\varepsilon)^{1 / 2}$; namely, we have $J_{e}^{\prime} J_{e}=\mathrm{id}$, $J_{e} J_{e}^{\prime}=\mathrm{id},\left\|J_{e}\right\| \leq 1,\left\|J_{e}^{\prime}\right\| \leq 1+\delta_{\varepsilon}$,

$$
\left\|J_{e}^{\prime}-J_{e}^{*}\right\| \leq \delta_{\varepsilon} \quad \text { and } \quad\left|\widetilde{\mathfrak{d}}_{\varepsilon}\left(J_{e} f, u\right)-\mathfrak{d}_{\varepsilon}\left(f, J_{e}^{\prime} u\right)\right| \leq \delta_{\varepsilon} .
$$

In particular, we get

$$
\left\|\left(\Delta_{\widetilde{X}_{\varepsilon, e}}+1\right)^{-1}-J_{e}\left(\Delta_{X_{\varepsilon, e}}+1\right)^{-1} J_{e}^{\prime}\right\| \leq 2 \delta_{\varepsilon}=\mathrm{O}(\varepsilon) .
$$

Before we check the closeness assumptions of [P06, Appendix] in the next section, we need some more notation and estimates. The estimates are already provided in EP05, P06], but we will also need a precise control of the edge length, when we approximate the $\delta_{\mathrm{s}}^{\prime}$-coupling by $\delta$-couplings in Section 4 below. Therefore, we present short proofs of the estimate here.

We first introduce the following averaging operators

$$
f_{v} u:=f_{X_{v}} u \mathrm{~d} x_{v} \quad \text { and } \quad f_{e} u(s):=f_{Y_{e}} u(s, \cdot) \mathrm{d} y_{e}
$$

for $u \in \mathrm{L}_{2}\left(X_{\varepsilon}\right)$, where we use the following symbols

$$
f_{X_{v}} u \mathrm{~d} x_{v}:=\frac{1}{\operatorname{vol}_{d} X_{v}} \int_{X_{v}} u \mathrm{~d} x_{v} \quad \text { and } \quad f_{Y_{e}} \varphi \mathrm{d} y_{e}:=\frac{1}{\operatorname{vol}_{d-1} Y_{e}} \int_{Y_{e}} \varphi \mathrm{d} y_{e}
$$

denoting the normalised integrals. For brevity, we also omit the measure and write $f_{\partial X_{v}} u$ etc.

In order to obtain the below Sobolev trace estimate (2.11), we need a further decomposition of the vertex neighbourhood $X_{v}$. Recall that $X_{v}$ has $(\operatorname{deg} v)$-many boundary components isometric to $Y_{e}$. We assume that each such boundary component has a collar neighbourhood $X_{v, e}=\left(0, \ell_{e}\right) \times Y_{e}$ of length $\ell_{e}$. Note that the scaled vertex neighbourhood $X_{\varepsilon, v}=\varepsilon X_{v}$ is of order $\varepsilon$ in all directions, so that the scaled collar neighbourhoods $X_{\varepsilon, v, e}:=\varepsilon X_{v, e}$ are of length $\varepsilon \ell_{e}$. We can always assume that such a decomposition exists, by possibly using a different cut of the manifold $X$ into $X_{v}$ and $X_{e}$, the price being an additional longitudinal error of order $\varepsilon$ (see Lemma 2.7). Similarly as in (2.3), one can get the following Sobolev trace estimates for the scaled manifolds:

$$
\begin{gathered}
\|u\|_{\partial_{e} X_{\varepsilon, v}}^{2} \leq \varepsilon \widetilde{a}\|\mathrm{~d} u\|_{X_{\varepsilon, v, e}}^{2}+\frac{2}{\varepsilon \widetilde{a}}\|u\|_{X_{\varepsilon, v, e}}^{2} \\
\|u\|_{\partial_{v} X_{\varepsilon, e}}^{2} \leq a\left\|u^{\prime}\right\|_{X_{\varepsilon, e}}^{2}+\frac{2}{a}\|u\|_{X_{\varepsilon, e}}^{2}
\end{gathered}
$$

for $0<a, \widetilde{a} \leq \ell_{e}$ on the vertex and edge neighbourhood, respectively, where $u^{\prime}=\partial_{s} u$ denotes the longitudinal derivative. The unscaled versions are obtained, of course, by 
APPROXIMATION OF VERTEX COUPLINGS BY SCHRÖDINGER OPERATORS ON MANIFOLDS 7

setting $\varepsilon=1$. Moreover, by the Cauchy-Schwarz inequality we get

$$
\operatorname{vol} Y_{e}\left|f_{e} u(0)\right|^{2} \leq\|u\|_{\partial_{e} X_{v}}^{2}=\|u\|_{\partial_{v} X_{e}}^{2} .
$$

In the following lemma we compare the averaging over the boundary of $X_{v}$ with the averaging over the whole space $X_{v}$ :

Lemma 2.8. For $u \in \mathrm{H}^{1}\left(X_{v}\right)$, we have

$$
\operatorname{vol} \partial X_{v}\left|f_{\partial X_{v}} u-f_{v} u\right|^{2} \leq \sum_{e \in E} \operatorname{vol} \partial_{e} X_{v}\left|f_{\partial_{e} X_{v}} u-f_{v} u\right|^{2} \leq\left(\widetilde{a}+\frac{2}{\widetilde{a} \lambda_{2}(v)}\right)\|\mathrm{d} u\|_{X_{v}}^{2}
$$

for $0<\widetilde{a} \leq \ell_{-}=\min _{e} \ell_{e}$, where $\lambda_{2}(v)$ denotes the second (i.e., first non-zero) eigenvalue of the Neumann Laplacian on $X_{v}$; the latter is defined conventionally as the operator associated to the form $\mathfrak{d}_{v}(u):=\|\mathrm{d} u\|_{X_{v}}^{2}$ with the domain $\operatorname{dom} \mathfrak{d}_{v}:=\mathrm{H}^{1}\left(X_{v}\right)$.

Proof. Using Cauchy-Schwarz twice and the estimate (2.11) for each edge $e$ and $\varepsilon=1$, we obtain

$$
\operatorname{vol} \partial X_{v}\left|f_{\partial X_{v}} w\right|^{2} \leq \sum_{e} \operatorname{vol} \partial_{e} X_{v}\left|f_{\partial_{e} X_{v}} w\right|^{2} \leq\|u\|_{\partial X_{v}}^{2} \leq \widetilde{a}\|\mathrm{~d} w\|_{X_{v}}^{2}+\frac{2}{\widetilde{a}}\|w\|_{X_{v}}^{2}
$$

for $0<\widetilde{a} \leq \ell_{-}$, using the fact that $\bigcup_{e} X_{v, e} \subset X_{v}$. We apply the above estimate to the function $w=P_{v} u:=u-f_{v} u$ and observe that

$$
\|w\|_{X_{v}}^{2} \leq \frac{1}{\lambda_{2}(v)}\|\mathrm{d} w\|_{X_{v}}^{2}
$$

as one can check using the fact that that $\mathrm{d} w=\mathrm{d} u$ and that $P_{v}$ is the projection onto the orthogonal complement of the first eigenfunction $\mathbb{1}_{v} \in \mathrm{L}_{2}\left(X_{v}\right)$.

We also need an estimate over the vertex neighbourhood. It will assure that in the limit $\varepsilon \rightarrow 0$, no family of normalised eigenfunctions $\left(u_{\varepsilon}\right)_{\varepsilon}$ with eigenvalues lying in a bounded interval can concentrate on $X_{\varepsilon, v}$.

Lemma 2.9. We have

$$
\|u\|_{X_{\varepsilon, v}}^{2} \leq 4 \varepsilon^{2}\left[\frac{1}{\lambda_{2}(v)}+c_{\mathrm{vol}}\left(\tilde{a}+\frac{2}{\widetilde{a} \lambda_{2}(v)}\right)\right]\|\mathrm{d} u\|_{X_{\varepsilon, v}}^{2}+4 \varepsilon c_{\mathrm{vol}}\left[a\left\|u^{\prime}\right\|_{X_{\varepsilon, E}}^{2}+\frac{2}{a}\|u\|_{X_{\varepsilon, E}}^{2}\right]
$$

for $0<a, \widetilde{a} \leq \ell_{-}=\min _{e} \ell_{e}$, where $c_{\mathrm{vol}}:=c_{\mathrm{vol}}(v)=\operatorname{vol} X_{v} / \operatorname{vol} \partial X_{v}$ and $X_{\varepsilon, E}:=\cup_{e} X_{\varepsilon, e}$ denotes the union of all edge neighbourhoods.

Proof. We start with the estimate

$$
\|u\|_{X_{\varepsilon, v}}^{2} \leq 2 \varepsilon^{d}\left(\left\|u-f_{v} u\right\|_{X_{v}}^{2}+\left\|f_{v} u\right\|_{X_{v}}^{2}\right) \leq 2 \varepsilon^{d}\left(\frac{2}{\lambda_{2}(v)}\|\mathrm{d} u\|_{X_{v}}^{2}+\operatorname{vol} X_{v}\left|f_{v} u\right|^{2}\right)
$$

using Lemma 2.8 and the fact that $f_{v} u$ is constant. Moreover, the last term can be estimated by

$$
\begin{aligned}
\operatorname{vol} \partial X_{v}\left|f_{v} u\right|^{2} & \leq 2 \operatorname{vol} \partial X_{v}\left(\left|f_{v} u-f_{\partial X_{v}} u\right|^{2}+\left|f_{\partial X_{v}} u\right|^{2}\right) \\
& \leq 2\left(\widetilde{a}+\frac{2}{\widetilde{a} \lambda_{2}(v)}\right)\|\mathrm{d} u\|_{X_{v}}^{2}+\sum_{e} \operatorname{vol} \partial_{e} X_{v}\left|f_{\partial_{e} X_{v}} u\right|^{2}
\end{aligned}
$$

using (2.13). Since $\partial_{e} X_{v}$ is isometric to $\partial_{v} X_{e}$, we can estimate the latter sum by

$$
\sum_{e} \operatorname{vol} \partial_{v} X_{e}\left|f_{\partial_{e} X_{v}} u\right|^{2} \leq \sum_{e}\|u\|_{\partial_{v} X_{e}}^{2} \leq a\left\|u^{\prime}\right\|_{X_{E}}^{2}+\frac{2}{a}\|u\|_{X_{E}}^{2}
$$

due to (2.12) for $\varepsilon=1$, each edge $e$ and $0<a \leq \ell_{-}$. Here, $X_{E}:=X_{1, E}$ is the union of the unscaled edge neighbourhoods. The desired estimate then follows from the scaling 
behaviour $\|\mathrm{d} u\|_{X_{\varepsilon, v}}^{2}=\varepsilon^{d-2}\|\mathrm{~d} u\|_{X_{v}}^{2}$ and $\|w\|_{X_{\varepsilon, e}}^{2}=\varepsilon^{d-1}\|w\|_{X_{e}}^{2}$ for $w=u$ or $w=u^{\prime}$ (where $u^{\prime}=\partial_{s} u$ denotes the longitudinal derivative).

We are now able to prove the relative (form-)boundedness of the Hamiltonian $H_{\varepsilon}$ with respect to the Laplacian $\Delta_{X_{\varepsilon}}$ for the indicated class of potentials. It is again important here to have a precise control of the constants $\varepsilon_{\eta}$ and $\widetilde{C}_{\eta}$ in terms of the various parameters of our spaces. This will be of particular importance when we deal with the approximation of the $\delta_{\mathrm{s}}^{\prime}$-coupling by $\delta$-couplings with shrinking spacing $a=\varepsilon^{\alpha}$ in Section 4 below.

Lemma 2.10. To a given $\eta \in(0,1)$ there exists $\varepsilon_{\eta}>0$ such that the form $\mathfrak{h}_{\varepsilon}$ is relatively form-bounded with respect to the free form $\mathfrak{d}_{\varepsilon}$ with relative bound $\eta$ for all $\varepsilon \in\left(0, \varepsilon_{\eta}\right]$, in other words, there exists $\widetilde{C}_{\eta}>0$ such that

$$
\left|\mathfrak{h}_{\varepsilon}(u)-\mathfrak{d}_{\varepsilon}(u)\right| \leq \eta \mathfrak{d}_{\varepsilon}(u)+\widetilde{C}_{\eta}\|u\|_{X_{\varepsilon}}^{2}
$$

whenever $0<\varepsilon \leq \varepsilon_{\eta}$, where the constants $\varepsilon_{\eta}$ and $\widetilde{C}_{\eta}$ are given by

$$
\begin{array}{r}
\varepsilon_{\eta}=\varepsilon_{\eta}\left(\|Q\|_{\infty}, \ell_{-}\right):=\frac{\eta}{4\|Q\|_{\infty}}\left[\frac{1}{\lambda_{2}(v)}+c_{\mathrm{vol}} \cdot\left(\ell_{-}+\frac{2}{\ell_{-} \lambda_{2}(v)}\right)\right]^{-1}, \\
\widetilde{C}_{\eta}=\widetilde{C}_{\eta}\left(\|Q\|_{\infty}, \ell_{-}\right):=8 c_{\mathrm{vol}}\|Q\|_{\infty} \max \left\{\frac{4 c_{\mathrm{vol}}\|Q\|_{\infty}}{\eta}, \frac{1}{\ell_{-}}\right\} .
\end{array}
$$

Note that $\varepsilon_{\eta}=\mathrm{O}\left(\ell_{-}\right)$and $\widetilde{C}_{\eta}=\mathrm{O}\left(\ell_{-}^{-1}\right)$ as $\ell_{-} \rightarrow 0$.

Proof. The potential $Q_{\varepsilon}=\varepsilon^{-1} Q$ is by assumption supported on the vertex neighbourhood $X_{v}$, therefore we have

$$
\begin{aligned}
& \mid \mathfrak{h}_{\varepsilon}(f)- \mathfrak{d}_{\varepsilon}(f) \mid \leq \frac{\|Q\|_{\infty}}{\varepsilon}\|u\|_{X_{\varepsilon, v}}^{2} \\
& \leq 4\|Q\|_{\infty}\left\{\varepsilon\left[\frac{1}{\lambda_{2}(v)}+c_{\mathrm{vol}} \cdot\left(\ell_{-}+\frac{2}{\ell_{-} \lambda_{2}(v)}\right)\right]\|\mathrm{d} u\|_{X_{\varepsilon, v}}^{2}+a c_{\mathrm{vol}}\left\|u^{\prime}\right\|_{X_{\varepsilon, E}}^{2}\right\} \\
&+\frac{8\|Q\|_{\infty} c_{\mathrm{vol}}}{a}\|u\|_{X_{\varepsilon, E}}^{2}
\end{aligned}
$$

using Lemma 2.9, for $0<a \leq \ell_{-}$and $\widetilde{a}:=\ell_{-}$. Choosing $a=\min \left\{\ell_{-}, \eta\left(4 c_{\mathrm{vol}}\|Q\|_{\infty}\right)^{-1}\right\}$ and $0<\varepsilon \leq \varepsilon_{\eta}$ with $\varepsilon_{\eta}$ as above, we can estimate the quadratic form contributions by

$$
\eta\left(\|\mathrm{d} u\|_{X_{\varepsilon, v}}^{2}+\left\|u^{\prime}\right\|_{X_{\varepsilon, E}}^{2}\right) \leq \eta\|\mathrm{d} u\|_{X_{\varepsilon}}^{2} .
$$

The expression for $\widetilde{C}_{\eta}$ then follows by evaluating the constant in front of the remaining norm.

We need to estimate the "free" quadratic form against the form associated with the Hamiltonian:

Corollary 2.11. The quadratic form $\mathfrak{h}_{\varepsilon}$ is closed. Moreover, setting $\eta=1 / 2$, we get the estimate

which holds provided $0<\varepsilon \leq \varepsilon_{1 / 2}$.

$$
\mathfrak{d}_{\varepsilon}(u) \leq 2\left(\mathfrak{h}_{\varepsilon}(u)+\widetilde{C}_{1 / 2}\|u\|_{X_{\varepsilon}}^{2}\right)
$$

As in Lemma 2.4, we can prove the following estimate in order to compare the "free" quadratic form with the graph norm of $H_{\varepsilon}$ :

Lemma 2.12. We have

$$
\|u\|_{\mathrm{H}^{1}\left(X_{\varepsilon}\right)}^{2}=\mathfrak{d}_{\varepsilon}(u)+\|u\|_{X_{\varepsilon}}^{2} \leq 2 \max \left\{\widetilde{C}_{1 / 2}, \sqrt{2}\right\}\left\|\left(H_{\varepsilon}-\mathrm{i}\right) u\right\|_{X_{\varepsilon}}^{2}
$$

for $u \in \operatorname{dom} H_{\varepsilon} \subset \operatorname{dom} \mathfrak{h}_{\varepsilon}=\mathrm{H}^{1}\left(X_{\varepsilon}\right)$ and $0<\varepsilon \leq \varepsilon_{0}$. 


\section{Approximation of $\delta$-COUplings}

After this preliminaries we can pass to our main problems. The first one concerns approximation of a $\delta$-coupling by Schrödinger operators with scaled potentials supported by the vertex regions. For the sake of simplicity most part of the discussion will be done for the situation with a single vertex as described in Section 2.1 .

3.1. Quasi-unitary operators. First we define quasi-unitary operators mapping from $\mathscr{H}$ to $\widetilde{\mathscr{H}}$ and vice versa, as well as their analogues on the scales of order one, namely $\mathscr{H}^{1}$ and $\widetilde{\mathscr{H}^{1}}$. Here,

$$
\mathscr{H}:=\mathrm{L}_{2}(G), \quad \mathscr{H}^{1}:=\mathrm{H}^{1}(G), \quad \widetilde{\mathscr{H}}:=\mathrm{L}_{2}\left(X_{\varepsilon}\right), \quad \widetilde{\mathscr{H}^{1}}:=\mathrm{H}^{1}\left(X_{\varepsilon}\right) .
$$

Moreover, we need a relation between the different constants of the graph and the manifold model introduced above. Specifically, we set

$$
p_{e}:=\left(\operatorname{vol}_{d-1} Y_{e}\right)^{1 / 2} \quad \text { and } \quad q(v)=\int_{X_{v}} Q \mathrm{~d} x_{v}
$$

Let $J: \mathscr{H} \longrightarrow \widetilde{\mathscr{H}}$ be given by

$$
J f:=\varepsilon^{-(d-1) / 2} \bigoplus_{e \in E}\left(f_{e} \otimes \mathbb{Z}_{e}\right) \oplus 0
$$

with respect to the decomposition (2.8). Here $\mathbb{\mathbb { H }}_{e}$ is the normalised eigenfunction of $Y_{e}$ associated to the lowest (zero) eigenvalue, i.e. $\mathbb{H}_{e}(y)=\left(\operatorname{vol}_{d-1} Y_{e}\right)^{-1 / 2}$. In order to relate the Sobolev spaces of order one we need a similar map: we define $J^{1}: \mathscr{H}^{1} \longrightarrow \widetilde{\mathscr{H}}^{1}$ by

$$
J^{1} f:=\varepsilon^{-(d-1) / 2}\left(\bigoplus_{e \in E}\left(f_{e} \otimes \mathbb{Z}_{e}\right) \oplus f(v) \mathbb{1}_{v}\right),
$$

where $\mathbb{1}_{v}$ is the constant function on $X_{v}$ with value 1 . Note that the latter operator is well defined:

$$
\left(J^{1} f\right)_{e}(0, y)=\varepsilon^{-(d-1) / 2} p_{e}^{-1} f_{e}(0)=\varepsilon^{-(d-1) / 2} f(v)=\left(J^{1} f\right)_{v}(x)
$$

for any $x \in X_{v}$ due to (3.2) and (2.2), i.e., the function $J^{1} f$ matches along the different components of the manifold, thus $J f \in \mathrm{H}^{1}\left(X_{\varepsilon}\right)$. Moreover, $f(v)$ is defined for $f \in \mathrm{H}^{1}(G)$ (see Lemma 2.1).

The mapping in the opposite direction, $J^{\prime}: \widetilde{\mathscr{H}} \longrightarrow \mathscr{H}$, is given by the adjoint, $J^{\prime}:=$ $J^{*}$, which means that

$$
\left(J^{\prime} u\right)_{e}(s)=\varepsilon^{(d-1) / 2}\left\langle\mathbb{Z}_{e}, u_{e}(s, \cdot)\right\rangle_{Y_{e}}=\varepsilon^{(d-1) / 2} p_{e} f_{e} u(s) .
$$

Furthermore, we define $J^{\prime 1}: \widetilde{\mathscr{H}^{1}} \longrightarrow \mathscr{H}^{1}$ by

$$
\left(J_{e}^{\prime 1} u\right)(s):=\varepsilon^{(d-1) / 2}\left[\left\langle\mathbb{\mathbb { H }}_{e}, u_{e}(s, \cdot)\right\rangle_{Y_{e}}+\chi_{e}(s) p_{e}\left(f_{v} u-f_{e} u(0)\right)\right] .
$$

Here $\chi_{e}$ is a smooth cut-off function such that $\chi_{e}(0)=1$ and $\chi_{e}\left(\ell_{e}\right)=0$. If we choose the function $\chi_{e}$ to be piecewise affine linear with $\chi_{e}(0)=1, \chi_{e}(a)=0$ and $\chi_{e}\left(\ell_{e}\right)=0$, then $\left\|\chi_{e}\right\|_{I_{e}}^{2}=a / 3 \leq a$ and $\left\|\chi_{e}^{\prime}\right\|_{I_{e}}^{2}=a^{-1}$. Moreover, $\left(J_{e}^{\prime 1} u\right)_{e}(0)=\varepsilon^{(d-1) / 2} p_{e} f_{v} u$ so that $f:=J_{e}^{\prime 1} u$ satisfies $\underline{f}(0) \in \mathbb{C} \underline{p}$, and therefore $f \in \mathrm{H}_{\underline{p}}^{1}(G)$. Note that by construction of the manifold, we have $f_{\partial e X_{v}} u=f_{e} u(0)$. 
3.2. Closeness assumptions. Let us start this subsection with a lower bound on the operators $H$ and $H_{\varepsilon}$ in terms of the model parameters; for the definitions of the constants $C_{1 / 2}, \varepsilon_{1 / 2}$ and $\widetilde{C}_{1 / 2}$ see Lemma 2.2 and Lemma 2.10. Note that $\widetilde{C}_{1 / 2}$ still depends on $\|Q\|_{\infty}$ and $\ell_{-}$.

Lemma 3.1. For $\varepsilon \in\left(0, \varepsilon_{1 / 2}\right]$ the operators $H_{\varepsilon}$ and $H$ are bounded from below by $\lambda_{0}:=$ $-\widetilde{C}_{1 / 2}$. Moreover, if all lengths are finite, i.e. $\ell_{e}<\infty$, and $q(v) \leq 0$, then we have

$$
\inf \sigma(H) \leq \frac{q(v)}{\operatorname{vol} X_{E}} \quad \text { and } \quad \inf \sigma\left(H_{\varepsilon}\right) \leq \frac{q(v)}{\operatorname{vol} X_{E}+\varepsilon \operatorname{vol} X_{v}},
$$

where $X_{E}:=\cup_{e} X_{e}$ is the union of the edge neighbourhoods.

Proof. We have to calculate the maximum of $C_{1 / 2}$ and $\widetilde{C}_{1 / 2}$. Due to (3.2) we have $|\underline{p}|^{2}=\operatorname{vol} \partial X_{v}$ and $|q(v)|=\left|\int_{X_{v}} Q \mathrm{~d} x_{v}\right| \leq\|Q\|_{\infty}$ vol $X_{v}$ so that

$$
C_{1 / 2} \leq \max \left\{4 c_{\mathrm{vol}}^{2}\|Q\|_{\infty}^{2}, \frac{2 c_{\mathrm{vol}}\|Q\|_{\infty}}{\ell_{-}}\right\} \leq \widetilde{C}_{1 / 2}=\max \left\{64 c_{\mathrm{vol}}^{2}\|Q\|_{\infty}^{2}, \frac{8 c_{\mathrm{vol}}\|Q\|_{\infty}}{\ell_{-}}\right\}
$$

where $c_{\mathrm{vol}}:=\operatorname{vol} X_{v} / \operatorname{vol} \partial X_{v}$. The spectral estimates then follow by inserting suitable test functions into the Rayleigh quotients $\mathfrak{h}(f) /\|f\|^{2}$ and $\mathfrak{h}_{\varepsilon}(u) /\|u\|^{2}$. For $f$, we choose the edgewise constant function $f_{e}(x)=p_{e}$. Note that $f \in \mathrm{H}_{\underline{p}}^{1}(G)$. On the manifold, we choose the constant $u:=J^{1} f=\varepsilon^{(d-1) / 2} \mathbb{1}$. The upper bound on the infimum on the spectrum follows by the relation $\ell_{e} p_{e}^{2}=\operatorname{vol} X_{e}$ using (3.2).

Now we are in position to demonstrate that the two Hamiltonians are close to each other. We start with estimates of the identification operators and the forms $\mathfrak{h}, \mathfrak{h}_{\varepsilon}$ in terms of the "free" quadratic forms $\mathfrak{d}$ and $\mathfrak{d}_{\varepsilon}$ :

Lemma 3.2. The identification operators $J, J^{\prime}=J^{*}, J^{1}, J^{\prime 1}$ and the quadratic forms $\mathfrak{h}_{\varepsilon}$ and $\mathfrak{h}$ fulfil the estimates

$$
\begin{array}{rlrl}
\left\|J f-J^{1} f\right\|^{2} & \leq \delta_{\varepsilon}^{2}\|f\|_{\mathrm{H}^{1}(G)}^{2}, & \left\|J^{\prime} u-J^{\prime 1} u\right\|^{2} & \leq \delta_{\varepsilon}^{2}\|u\|_{\mathrm{H}^{1}\left(X_{\varepsilon}\right)}^{2}, \\
\|J f\|^{2} & =\|f\|^{2}, & \left\|J^{\prime} u\right\|^{2} \leq\|u\|^{2}, \\
J^{\prime} J f & =f, & \left\|J J^{\prime} u-u\right\|^{2} \leq \delta_{\varepsilon}^{2}\|u\|_{\mathrm{H}^{1}\left(X_{\varepsilon}\right)}^{2}, \\
\left|\mathfrak{h}\left(J^{\prime 1} u, f\right)-\mathfrak{h}_{\varepsilon}\left(u, J^{1} f\right)\right| & \leq \delta_{\varepsilon}\|u\|_{\mathrm{H}^{1}\left(X_{\varepsilon}\right)}\|f\|_{\mathrm{H}^{1}(G)} & &
\end{array}
$$

with $\delta_{\varepsilon}=\mathrm{O}\left(\varepsilon^{1 / 2}\right)$ as $\varepsilon \rightarrow 0$, being given explicitly by

$$
\begin{aligned}
\delta_{\varepsilon}^{2}:=\max \left\{\frac{8 \varepsilon c_{\mathrm{vol}}}{\ell_{0}}, \frac{\varepsilon^{2}}{\lambda_{2}(E)}, 4 \varepsilon^{2}\left[\frac{1}{\lambda_{2}(v)}+c_{\mathrm{vol}}\left(1+\frac{2}{\ell_{0} \lambda_{2}(v)}\right)\right],\right. & \\
& \left.\frac{2 \varepsilon}{\ell_{0}}\left(1+\frac{2}{\ell_{0} \lambda_{2}(v)}\right), \frac{4 \varepsilon c_{\mathrm{vol}}\|Q\|_{\infty}^{2}}{\ell_{0} \lambda_{2}(v)}\right\} .
\end{aligned}
$$

Here, $\ell_{0}:=\min \left\{1, \ell_{-}\right\}=\min _{e}\left\{1, \ell_{e}\right\} \leq 1, \lambda_{2}(E):=\min _{e} \lambda_{2}(e)$ and $c_{\mathrm{vol}}=\operatorname{vol} X_{v} / \operatorname{vol} \partial X_{v}$. Moreover, $\lambda_{2}(e)$ and $\lambda_{2}(v)$ denote the second (first non-vanishing) eigenvalue of the (Neumann-)Laplacian on $Y_{e}$ and $X_{v}$, respectively.

Proof. The first condition in (3.8a) is here

$$
\left\|J f-J^{1} f\right\|_{X_{\varepsilon}}^{2}=\varepsilon \operatorname{vol} X_{v}|f(v)|^{2} \leq \varepsilon c_{\mathrm{vol}}\left(\left\|f^{\prime}\right\|_{G}^{2}+\frac{2}{\ell_{0}}\|f\|_{G}^{2}\right)
$$

using Lemma 2.1 with $a=\ell_{0}$ and the fact that $|\underline{p}|^{2}=\operatorname{vol} \partial X_{v}$ due to (3.2). Next we need to show the second estimate in (3.8a). In our situation, we have

$$
\left\|J^{\prime} u-J^{\prime 1} u\right\|_{G}^{2}=\varepsilon^{d-1} \sum_{e \in E}\left\|\chi_{e}\right\|_{I_{e}}^{2} p_{e}^{2}\left|f_{v} u-f_{e} u(0)\right|^{2} \leq \varepsilon\left(1+\frac{2}{\ell_{0} \lambda_{2}(v)}\right)\|\mathrm{d} u\|_{X_{\varepsilon, v}}^{2}
$$


APPROXIMATION OF VERTEX COUPLINGS BY SCHRÖDINGER OPERATORS ON MANIFOLDSI

using Lemma 2.8 with $a=\widetilde{a}=\ell_{0}$. Moreover, (3.8b) and the first equation in (3.8c) are easily seen to be fulfilled. The second estimate in (3.8c) is more involved. Here, we have

$$
\left\|J J^{\prime} u-u\right\|^{2}=\sum_{e}\left\|u-f_{e} u\right\|_{X_{\varepsilon, e}}^{2}+\|u\|_{X_{\varepsilon, v}}^{2} .
$$

The first term can be estimated as in (2.14) by

$$
\left\|u-f_{e} u\right\|_{X_{\varepsilon, e}}^{2}=\int_{I_{e}}\left\|u(s)-f_{e} u(s)\right\|_{Y_{e}}^{2} \mathrm{~d} s \leq \frac{1}{\lambda_{2}(e)} \int_{I_{e}}\left\|\mathrm{~d}_{Y_{e}} u(s)\right\|_{Y_{e}}^{2} \mathrm{~d} s=\frac{\varepsilon^{2}}{\lambda_{2}(e)}\left\|\mathrm{d}_{Y_{e}} u\right\|_{X_{\varepsilon, e}}^{2},
$$

where $u(s):=u(s, \cdot)$. The second term can be estimated by Lemma 2.9, so that

$$
\delta_{\varepsilon}^{2} \geq \max \left\{4 \varepsilon^{2}\left[\frac{1}{\lambda_{2}(v)}+c_{\mathrm{vol}}\left(1+\frac{2}{\ell_{0} \lambda_{2}(v)}\right)\right], \frac{\varepsilon^{2}}{\lambda_{2}(E)}, \frac{8 \varepsilon c_{\mathrm{vol}}}{\ell_{0}}\right\}
$$

which is sufficient for the estimate (3.8c).

Let us finally prove (3.8d $)$ in our model. Note that this estimate differs from the ones given in [P06] by the absence of the potential term $Q_{\varepsilon}=\varepsilon^{-1} Q$ there. In our situation, we have

$$
\begin{aligned}
\mid \mathfrak{h}\left(J^{\prime 1} u\right. & , f)-\left.\mathfrak{h}_{\varepsilon}\left(u, J^{1} f\right)\right|^{2} \\
& \leq 2 \varepsilon^{d-1}\left[\left|\sum_{e} p_{e}\left(f_{v} \bar{u}-f_{e} \bar{u}(0)\right)\left\langle\chi_{e}^{\prime}, f^{\prime}\right\rangle_{I_{e}}\right|^{2}+\left|q(v) f_{v} \bar{u}-\left\langle Q u, \mathbb{1}_{v}\right\rangle_{X_{v}}\right|^{2}|f(v)|^{2}\right] .
\end{aligned}
$$

Note that the derivative terms cancel on the edges due to the product structure of the metric and the fact that $\mathrm{d}_{Y_{e}} \mathbb{\mathbb { H }}_{e}=0$ and the vertex contribution vanishes due to $\mathrm{d}_{X_{v}} \mathbb{1}=0$. The first term can be estimated by

$$
2 \varepsilon\left(\widetilde{a}+\frac{2}{\widetilde{a} \lambda_{2}(v)}\right) \frac{1}{a}\|\mathrm{~d} u\|_{X_{\varepsilon, v}}^{2} \leq \frac{2 \varepsilon}{\ell_{0}}\left(1+\frac{2}{\ell_{0} \lambda_{2}(v)}\right)
$$

using Cauchy-Schwarz, Lemma 2.8 and the fact that $\left\|\chi_{e}^{\prime}\right\|_{I_{e}}^{2}=1 / a \leq 1 / \ell_{0}$ by our choice of $\chi_{e}$. For the second term, we use our definition $q(v)=\int_{X_{v}} Q \mathrm{~d} x_{v}$ and $q(v) f_{v} \bar{u}=$ $\left\langle u, f_{v} Q \mathbb{1}_{v}\right\rangle_{X_{v}}$ to conclude

$$
\begin{aligned}
\left|q(v) f_{v} \bar{u}-\left\langle Q u, \mathbb{1}_{v}\right\rangle_{X_{v}}\right|^{2}=\left|\left\langle u, f_{v} Q-Q\right\rangle_{X_{v}}\right|^{2} & \\
& =\left|\left\langle u, P_{v} Q\right\rangle_{X_{v}}\right|^{2}=\left|\left\langle P_{v} u, Q\right\rangle_{X_{v}}\right|^{2} \leq \frac{1}{\lambda_{2}(v)}\|\mathrm{d} u\|_{X_{v}}^{2}\|Q\|_{X_{v}}^{2}
\end{aligned}
$$

where $P_{v} u:=u-f_{v} u$ is the projection onto the orthogonal complement of $\mathbb{1}_{v}$. The last estimate follows from (2.14). Collecting the error terms for the sesquilinear form estimate, we obtain

$$
\delta_{\varepsilon}^{2} \geq \max \left\{\frac{2 \varepsilon}{\ell_{0}}\left(1+\frac{2}{\ell_{0} \lambda_{2}(v)}\right), \frac{4 \varepsilon c_{\mathrm{vol}}\|Q\|_{\infty}^{2}}{\ell_{0} \lambda_{2}(v)}\right\}
$$

as lower bound on $\delta_{\varepsilon}$, using also Lemma 2.1 for the estimate on $|f(v)|^{2}$, and $\|Q\|_{X_{v}}^{2} \leq$ $\operatorname{vol} X_{v}\|Q\|_{\infty}^{2}$.

Now we can prove our main result on the approximation of a $\delta$-coupling in the manifold model; for more details on the notion of " $\delta$-closeness" we refer to [P06, App.]. The resolvent estimate at $z=\mathrm{i}$ will be needed in Section 4 when the lower bound $\lambda_{0}$ depends on $\varepsilon$ and may tend to $-\infty$ as $\varepsilon \rightarrow 0$. Recall the definition of $\widetilde{C}_{1 / 2}, 0<\varepsilon_{1 / 2}($ see $(2.15))$ and $\lambda_{0}:=-\widetilde{C}_{1 / 2}$, and that $\widetilde{C}_{1 / 2} \geq C_{1 / 2}$. 
Theorem 3.3. For $\varepsilon \in\left(0, \varepsilon_{1 / 2}\right]$, the operators $H_{\varepsilon}-\lambda_{0}$ and $H-\lambda_{0}$ are $\sqrt{2} \delta_{\varepsilon}$-close with $\delta_{\varepsilon}=\mathrm{O}\left(\varepsilon^{1 / 2}\right)$ given in (3.9); in other words, there is an identification operator $J: \mathrm{L}_{2}(G) \longrightarrow \mathrm{L}_{2}\left(X_{\varepsilon}\right)$ such that $J^{*} J=\mathrm{id}$,

$$
\left\|\left(\mathrm{id}-J J^{*}\right)\left(H_{\varepsilon}-\lambda_{0}\right)^{-1}\right\| \leq \sqrt{2} \delta_{\varepsilon} \quad \text { and } \quad\left\|J\left(H-\lambda_{0}\right)^{-1}-\left(H_{\varepsilon}-\lambda_{0}\right)^{-1} J\right\| \leq 3 \sqrt{2} \delta_{\varepsilon} .
$$

Moreover, for $\varepsilon \in\left(0, \varepsilon_{1 / 2}\right]$ we have the estimate

$$
\left\|J(H-\mathrm{i})^{-1}-\left(H_{\varepsilon}-\mathrm{i}\right)^{-1} J\right\| \leq 10 \delta_{\varepsilon} \max \left\{\widetilde{C}_{1 / 2}, \sqrt{2}\right\},
$$

where $\|\cdot\|$ denotes the operator norm for operators from $\mathrm{L}_{2}(G)$ into $\mathrm{L}_{2}\left(X_{\varepsilon}\right)$.

Proof. The closeness of the operators $H-\lambda_{0}$ and $H_{\varepsilon}-\lambda_{0}$ follows from the estimate

$$
\|\mathrm{d} u\|_{X_{\varepsilon}}^{2}+\|u\|_{X_{\varepsilon}}^{2} \leq 2\left(\mathfrak{h}_{\varepsilon}(u)+\left(1-\lambda_{0}\right)\|u\|_{X_{\varepsilon}}^{2}\right)=2\left\|\left(H_{\varepsilon}-\lambda_{0}+1\right)^{1 / 2} u\right\|_{X_{\varepsilon}}^{2}
$$

by Corollary 2.11, and similarly for $H$ on $G$ by Corollary [2.3 and (3.7), together with Lemma 3.2. The resolvent estimate can be seen as follows: Let $R:=(H-\mathrm{i})^{-1}$ and $R:=\left(H_{\varepsilon}-\mathrm{i}\right)^{-1}$, and let $\tilde{f} \in \mathrm{L}_{2}(G), \widetilde{u} \in \mathrm{L}_{2}\left(X_{\varepsilon}\right)$. Setting $f:=R \tilde{f} \in \operatorname{dom} H$ and $u:=R_{\varepsilon} \widetilde{u} \in \operatorname{dom} H_{\varepsilon}$, we have

$$
\begin{aligned}
&\left\langle\widetilde{u},\left(J R-R_{\varepsilon} J\right) \widetilde{f}\right\rangle=\langle\widetilde{u}, J f\rangle-\langle u, J \widetilde{f}\rangle \\
&=\left\langle\widetilde{u},\left(J-J^{1}\right) f\right\rangle+\left(\mathfrak{h}_{\varepsilon}\left(u, J^{1} f\right)-\mathfrak{h}\left(J^{\prime 1} u, f\right)\right)+\left\langle\left(J^{\prime 1}-J^{*}\right) u, \widetilde{f}\right\rangle \\
&-\mathrm{i}\left(\left\langle u,\left(J^{1}-J\right) f\right\rangle+\left\langle\left(J^{\prime 1}-J^{*}\right) u, f\right\rangle\right),
\end{aligned}
$$

and therefore

$$
\left|\left\langle\widetilde{u},\left(J R-R_{\varepsilon} J\right) \widetilde{f}\right\rangle\right| \leq 10 \delta_{\varepsilon} \max \left\{\widetilde{C}_{1 / 2}, \sqrt{2}\right\}\|\widetilde{f}\|\|\widetilde{u}\|
$$

using Lemmata 2.4 and 2.12 , and the fact that $C_{1 / 2} \leq \widetilde{C}_{1 / 2}$.

Using the abstract results of [P06, App. A] or [P08], we can show the resolvent convergence and the convergence other functions of the operator:

Theorem 3.4. We have

$$
\begin{aligned}
& \left\|J(H-z)^{-1}-\left(H_{\varepsilon}-z\right)^{-1} J\right\|=\mathrm{O}\left(\varepsilon^{1 / 2}\right), \\
& \left\|J(H-z)^{-1} J^{\prime}-\left(H_{\varepsilon}-z\right)^{-1}\right\|=\mathrm{O}\left(\varepsilon^{1 / 2}\right)
\end{aligned}
$$

for $z \notin\left[\lambda_{0}, \infty\right)$. The error depends only on $\delta_{\varepsilon}$, given in (3.9), and on $z$. Moreover, we can replace the function $\varphi(\lambda)=(\lambda-z)^{-1}$ by any measurable, bounded function converging to a constant as $\lambda \rightarrow \infty$ and being continuous in a neighbourhood of $\sigma(H)$.

The following spectral convergence is also a consequence of the $\mathrm{O}\left(\varepsilon^{1 / 2}\right)$-closeness; for details of the uniform convergence of sets, i.e. the convergence in Hausdorff-distance sense we refer to [HN99, App. A] or [P08].

Theorem 3.5. The spectrum of $H_{\varepsilon}$ converges to the spectrum of $H$ uniformly on any finite energy interval. The same is true for the essential spectrum.

Proof. The spectral convergence is a direct consequence of the closeness, as it follows from the general theory developed in [P06, Appendix] and [P08].

For the discrete spectrum we have the following result:

Theorem 3.6. For any $\lambda \in \sigma_{\text {disc }}(H)$ there exists a family $\left\{\lambda_{\varepsilon}\right\}_{\varepsilon}$ with $\lambda_{\varepsilon} \in \sigma_{\text {disc }}\left(H_{\varepsilon}\right)$ such that $\lambda_{\varepsilon} \rightarrow \lambda$ as $\varepsilon \rightarrow 0$. Moreover, the multiplicity is preserved. If $\lambda$ is a simple eigenvalue with normalised eigenfunction $\varphi$, then there exists a family of simple normalised eigenfunctions $\left\{\varphi_{\varepsilon}\right\}_{\varepsilon}$ of $H_{\varepsilon}$ ( $\varepsilon$ small) such that

$$
\left\|J \varphi-\varphi_{\varepsilon}\right\|_{X_{\varepsilon}} \rightarrow 0
$$

as $\varepsilon \rightarrow 0$. 
We remark that the convergence of higher-dimensional eigenspaces is also valid, however, it requires some technicalities which we skip here.

To summarise, we have shown that the $\delta$-coupling with weighted entries can be approximated by a geometric setting and a potential located on the vertex neighbourhood.

Let us briefly sketch how to extend the above convergence results Theorems 3.3 to more complicated - even to non-compact — graphs. Denote by $G$ a metric graph, given by the underlying discrete graph $(V, E, \partial)$ with $\partial: E \longrightarrow V \times V, \partial e=\left(\partial_{-} e, \partial_{+} e\right)$ denoting the initial and terminal vertex, and the length function $\ell: E \longrightarrow(0, \infty)$, such that each edge $e$ is identified with the interval $I_{e}=\left(0, \ell_{e}\right)$ (for simplicity, we assume here that all length are finite, i.e., $\left.\ell_{e}<\infty\right)$. Let $X_{\varepsilon}$ be the corresponding approximating manifold constructed from the building blocks $X_{\varepsilon, e}=I_{e} \times \varepsilon Y_{e}$ and $X_{\varepsilon, v}=\varepsilon X_{v}$ as in Section 2.2. For more details, we refer to [EP05, P06, EP08, P08]. Since a metric graph can be constructed from a number of star graphs with identified end points of the free ends, we can define global identification operators. We only have to assure that the global error we make is still uniformly bounded:

Theorem 3.7. Assume that $G$ is a metric graph and $X_{\varepsilon}$ the corresponding approximating manifold constructed according to $G$. If

$$
\inf _{v \in V} \lambda_{2}(v)>0, \quad \sup _{v \in V} \frac{\operatorname{vol} X_{v}}{\operatorname{vol} \partial X_{v}}<\infty, \quad \sup _{v \in V}\left\|Q \uparrow_{X_{v}}\right\|_{\infty}<\infty, \quad \inf _{e \in E} \lambda_{2}(e)>0, \quad \inf _{e \in E} \ell_{e}>0,
$$

then the corresponding Hamiltonians $H=\Delta_{G}+\sum_{v} q(v) \delta_{v}$ and $H_{\varepsilon}=\Delta_{X_{\varepsilon}}+\sum_{v} \varepsilon^{-1} Q_{v}$ are $\delta_{\varepsilon}$-close, where the error $\delta_{\varepsilon}=\mathrm{O}\left(\varepsilon^{1 / 2}\right)$ depends only on the above indicated global constants.

\section{Approximation of the $\delta_{\mathrm{s}}^{\prime}$-COUplings}

The main aim of this section is to show how a the symmetrised $\delta^{\prime}$-coupling, or $\delta_{\mathrm{s}}^{\prime}$, can be approximated using manifold model discussed above. To this aim we shall use a result of [CE04 by which a $\delta_{\mathrm{s}}^{\prime}$-coupling can be approximated by means of several $\delta$-couplings on the same metric graph, located close to the vertex and "lift" this approximation to the manifold. For the sake simplicity we will again consider the star-shape setting with a single vertex. We want to stress, however, that the method we use can be directly generalised to more complicated graphs but also, what is equally important, to other vertex couplings, once they can be approximated by combinations of $\delta$-couplings on the graph, possibly with an addition of extra edges — see [ET06, ET07].

Let thus $G=I_{v_{0}}$ be a star graph as in Section 2 where we denote the vertex in the centre by $v_{0}$ and where we label the $n=\operatorname{deg} v$ edges by $e=1, \ldots, n$. Again for simplicity, we assume that all the (unscaled) transversal volumes $p_{e}^{2}=\operatorname{vol} Y_{e}$ are the same; without loss of generality we may put vol $Y_{e}=1$. Moreover, we assume that all lengths are finite, i.e. $\ell_{e}<\infty$, and equal, so we may put $\ell_{e}=1$. First we recall the definition of the $\delta_{\mathrm{s}}^{\prime}-$ coupling: the operator $H^{\beta}$, formally written as $H^{\beta}=\Delta_{G}+\beta \delta_{v_{0}}^{\prime}$, acts as $\left(H^{\beta} f\right)_{e}=-f_{e}^{\prime \prime}$ on each edge for functions $f$ in the domain

$$
\begin{array}{r}
\operatorname{dom} H^{\beta}:=\left\{f \in \mathrm{H}_{\max }^{2}(G) \mid \forall e_{1}, e_{2}: f_{e_{1}}^{\prime}(0)=f_{e_{2}}^{\prime}(0)=: f^{\prime}(0), \sum_{e} f_{e}(0)=\beta f^{\prime}(0),\right. \\
\left.\forall e: f_{e}^{\prime}\left(\ell_{e}\right)=0\right\} .
\end{array}
$$

For the sake of definiteness we imposed here Neumann conditions at the free ends of the edges, however, the choice is not substantial; we could use equally well Dirichlet or any 
other boundary condition. The corresponding quadratic form is given as

$$
\mathfrak{h}^{\beta}(f)=\sum_{e}\left\|f_{e}^{\prime}\right\|^{2}+\frac{1}{\beta}\left|\sum_{e} f_{e}(0)\right|^{2}, \quad \operatorname{dom} \mathfrak{h}^{\beta}=\mathrm{H}_{\max }^{1}(G)
$$

if $\beta \neq 0$ and

$$
\mathfrak{h}^{\beta}(f)=\sum_{e}\left\|f_{e}^{\prime}\right\|^{2}, \quad \operatorname{dom} \mathfrak{h}^{\beta}=\left\{f \in \mathrm{H}_{\max }^{1}(G) \mid \sum_{e} f_{e}(0)=0\right\}
$$

if $\beta=0$; the condition $f \in H^{0}$ is obviously dual to the free (or Kirchhoff) vertex coupling - see, e.g., [Ku04, Sec. 3.2.3].

The (negative) spectrum of $H^{\beta}$ is easily found:

Lemma 4.1. If $\beta \geq 0$ then $H^{\beta} \geq 0$. On the other hand, if $\beta<0$ then $H^{\beta}$ has exactly one negative eigenvalue $\lambda=-\kappa^{2}$ where $\kappa$ is the solution of the equation

$$
\cosh \kappa+\frac{\beta \kappa}{\operatorname{deg} v} \sinh \kappa=0 .
$$

Proof. The non-negativity of $H^{\beta}$ follows from the quadratic form expression for $\beta>0$ and $\beta=0$. We make the ansatz

$$
f_{e}(s)=\cosh \kappa(1-s)
$$

fulfilling automatically the Neumann condition at $s=1$ and the continuity condition at $s=0$ since $f_{e}^{\prime}(0)=-\kappa \sinh \kappa$ is independent of $e$. The remaining condition at zero leads to the above relation of $\kappa$ and $\beta$, showing in another way that if $\beta \geq 0$ there cannot exist a negative eigenvalue.

The main idea of the approximation of a $\delta_{\mathrm{s}}^{\prime}$-coupling by Schrödinger operators on a manifold is to employ a combination of $\delta$-couplings in an operator one may call an intermediate Hamiltonian $H^{\beta, a}$, and then to use the approximations for $\delta$-couplings given in the previous section.

In order to define $H^{\beta, a}$, we first modify the (discrete) structure of the graph $G$ inserting additional vertices $v_{e}$ of degree 2 on the edge $e$ with the distance $a \in(0,1)$ from the central vertex $v_{0}$ (see Figure 1). Each edge $e$ is splitted into two edges $e_{a}$ and $e_{1}$. We denote the metric graph with the additional vertices $v_{e}$ and splitted edges by $G_{a}$, i.e., $V\left(G_{a}\right)=\left\{v_{0}\right\} \cup\left\{v_{e} \mid e=1, \ldots, n\right\}, E\left(G_{a}\right)=\left\{e_{a}, e_{1} \mid e=1, \ldots n\right\}$ and $\ell_{e_{a}}=a$, $\ell_{e_{1}}=1-a$. This metrically equivalent graph $G_{a}$ will be needed when associating the corresponding manifold.

Remark 4.2. It is useful to note that the Laplacians $\Delta_{G}$ and $\Delta_{G_{a}}$ associated to the metric graphs $G$ and $G_{a}$ are unitarily equivalent. Indeed, introducing additional vertices of degree two does not change the original quadratic form $\mathfrak{d}_{G}$ with the domain $\mathrm{H}^{1}(G)=$ dom $\mathfrak{d}$ associated to the free operator $\Delta_{G}=H_{(G, 0)}$. Figuratively speaking, the free operator does not see these vertices of degree two. We just have to change the coordinate on the edge $e$, i.e. we can either use the original coordinate $s \in\left(0, \ell_{e}\right)$ on the edge $e$ or we can split the edge $e$ into two edges $e_{a}$ and $e_{1}$ of length $\ell_{e_{a}}=a$ and $\ell_{e_{1}}=\ell_{e}-a=1-a$ with the corresponding coordinates.

The core of the approximation lies in a suitable, $a$-dependent choice of the parameters of these $\delta$-couplings. Writing the operator in terms of the formal notation introduced in (2.6), we put

$$
H^{\beta, a}:=\Delta_{G}+b(a) \delta_{v_{0}}+\sum_{e} c(a) \delta_{v_{e}}, \quad b(a)=-\frac{\beta}{a^{2}}, \quad c(a)=-\frac{1}{a},
$$


to be the intermediate Hamiltonian. Notice that the strength of central $\delta$-coupling depends on $\beta$ while the added $\delta$-interactions are attractive, the sole parameter being the distance $a$. The operator can be defined via its quadratic form

$$
\mathfrak{h}^{\beta, a}(f):=\sum_{e}\left\|f_{e}^{\prime}\right\|^{2}-\frac{\beta}{a^{2}} f(0)-\frac{1}{a} \sum_{e}\left|f_{e}(a)\right|^{2}, \quad \operatorname{dom} \mathfrak{h}^{a}=\mathrm{H}^{1}(G),
$$

where $\mathrm{H}^{1}(G)=\mathrm{H}_{\underline{p}}^{1}(G)$ with $\underline{p}=(1, \ldots, 1)$, i.e. the functions $f \in \mathrm{H}^{1}(G)$ are distinguished by being continuous at $v_{0}, f_{e_{1}}(0)=f_{e_{2}}(0)=: f(0)$.

The next theorem shows that the intermediate Hamiltonian converges indeed to the $\delta_{\mathrm{s}}^{\prime}$-coupling with the strength $\beta$ on the star-shaped graph:

Theorem 4.3 (Cheon, Exner). We have

$$
\left\|\left(H^{\beta, a}-z\right)^{-1}-\left(H^{\beta}-z\right)^{-1}\right\|=\mathrm{O}(a)
$$

as $a \rightarrow 0$ for $z \notin \mathbb{R}$, where $\|\cdot\|$ denotes the operator norm on $\mathrm{L}_{2}(G) .3$

Note that the choice of the parameters $b(a) c(a)$ of the $\delta$-interactions as functions of the distance $a$ follows from a careful analysis of the resolvents of $H^{\beta, a}$ and $H^{\beta}$. Each of these is highly singular as $a \rightarrow 0$, however, in the difference all the singularities cancel leaving us with a vanishing expression. Needless to say, that such a limiting process is highly non-generic.

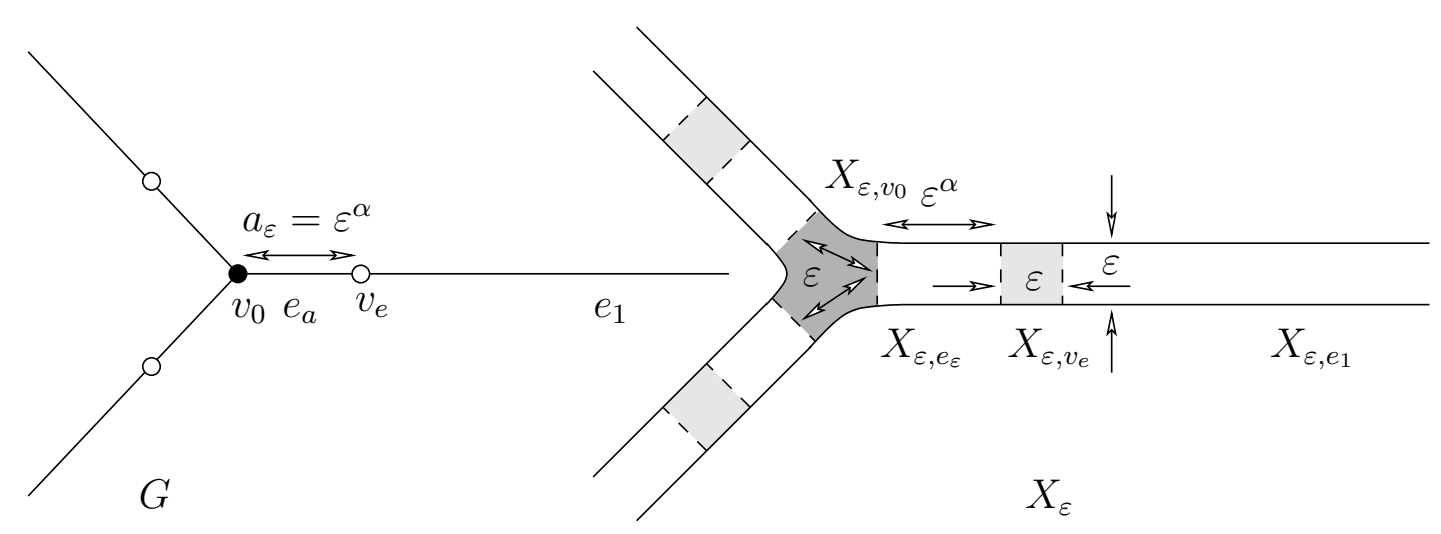

FIGURE 1. The intermediate graph picture used in the $\delta_{\mathrm{s}}^{\prime}$-approximation and the corresponding manifold model.

Let us now consider the manifold model approaching the intermediate situation Hamiltonian $H^{\beta, a}$ in the limit $\varepsilon \rightarrow 0$ with $a=a_{\varepsilon}=\varepsilon^{\alpha}$ and $0<\alpha<1$ to be specified later on. Let $X_{\varepsilon}$ be a manifold model of the graph $G$ as shown in Figure 1. For the additional vertices of degree two we choose the vertex neighbourhoods as a part of the cylinder of length $\varepsilon$ and distance of order of $a_{\varepsilon}$ from the central vertex $v_{0}$. The edge $e_{a_{\varepsilon}}=: e_{\varepsilon}$ now has the length $a_{\varepsilon}=\varepsilon^{\alpha}$ depending on $\varepsilon$. The "free" edge $e_{1}$ joining $v_{e}$ with the free end point at $s=1$ is again $\varepsilon$-depending, namely it has the length $1-a_{\varepsilon}=1-\varepsilon^{\alpha}$. By the argument given in Lemma 2.7 we can deal with this error and assume that this edge again has length one, the price being an extra error of order $\mathrm{O}\left(\varepsilon^{\alpha}\right)$, affecting neither the final result nor the quantitative error estimate. Next we have to choose the potentials

\footnotetext{
${ }^{3}$ The claim made in [CE04 is only that the norm tends to zero, however, the rate with which it vanishes is obvious from the proof. We remove the superfluous $\operatorname{deg} v$ from the definition of $H^{\beta, a}$ in that paper. It should also be noted that the proof in [CE04] is given for star graphs with semi-infinite edges but the argument again modifies easily to the finite-length situation we consider for convenience here.
} 
in the vicinity of the vertices $v=v_{0}$ and $v=v_{e}$. The simplest option is to assume that they are constant,

$$
Q_{\varepsilon, v}(x):=\frac{1}{\varepsilon} \cdot \frac{q_{\varepsilon}(v)}{\operatorname{vol} X_{v}}, \quad x \in X_{v}
$$

so that $\int_{X_{v}} Q_{\varepsilon, v} \mathrm{~d} x=\varepsilon^{-1} q_{\varepsilon}(v)$ (see (2.10) ) and (3.2) $)$, where we put

$$
q_{\varepsilon}\left(v_{0}\right):=b\left(\varepsilon^{\alpha}\right)=-\beta \varepsilon^{-2 \alpha} \quad \text { and } \quad q_{\varepsilon}\left(v_{e}\right):=c\left(\varepsilon^{\alpha}\right)=-\varepsilon^{-\alpha} .
$$

The corresponding manifold Hamiltonian and the respective quadratic form are then given by

$$
H_{\varepsilon}^{\beta}=\Delta_{X_{\varepsilon}}-\varepsilon^{-1-2 \alpha} \frac{\beta}{\operatorname{vol} X_{v_{0}}} \mathbb{1}_{X_{v_{0}}}-\varepsilon^{-1-\alpha} \sum_{e \in E} \mathbb{1}_{X_{v_{e}}}
$$

and

$$
\mathfrak{h}_{\varepsilon}^{\beta}(u)=\|\mathrm{d} u\|_{X_{\varepsilon}}^{2}-\varepsilon^{-1-2 \alpha} \frac{\beta}{\operatorname{vol} X_{v_{0}}}\|u\|_{X_{\varepsilon, v_{0}}}^{2}-\varepsilon^{-1-\alpha} \sum_{e \in E}\|u\|_{X_{\varepsilon, v_{e}}}^{2},
$$

respectively. Note that the unscaled vertex neighbourhood $X_{v_{e}}$ of the added vertex $v_{e}$ has volume 1 by construction.

Before proceeding to the approximation itself, let us first make some comments about the lower bounds of the operators $H^{\beta, a}$ and their manifold approximations $H_{\varepsilon}^{\beta}$ :

Lemma 4.4. If $\beta<0$, then the spectrum of $H^{\beta, a}$ is uniformly bounded from below as $a \rightarrow 0$, in other words, there is a constant $C>0$ such that

$$
\inf \sigma\left(H^{\beta, a}\right) \geq-C \text { as } a \rightarrow 0 .
$$

If $\beta \geq 0$, on the other hand, then the spectrum of $H^{\beta, a}$ is asymptotically unbounded from below,

$$
\inf \sigma\left(H^{\beta, a}\right) \rightarrow-\infty \quad \text { as } \quad a \rightarrow 0 .
$$

Note that although we know the limit spectrum as $a \rightarrow 0$ (see Lemma 4.1), the resolvent convergence of Theorem 4.3 does not necessarily imply the uniform boundedness from below of $H^{\beta, a}$ (see Remark 4.8).

Proof. Let $\beta<0$. Then an eigenfunction on the (original) edge $e$ has the form

$$
f_{e}(s)= \begin{cases}A \cosh (\kappa s)+B_{e} \sinh (\kappa s), & 0 \leq s \leq a \\ C_{e} \cosh (\kappa(1-s)), & a \leq s \leq 1 .\end{cases}
$$

for $\kappa>0$, the corresponding eigenvalue being $\lambda=-\kappa^{2}$. The Neumann condition $f_{e}^{\prime}(1)=$ 0 at $s=1$ is automatically fulfilled, as well as the continuity at $s=0$ for the different edges $e$, since $f_{e}(0)=A$ is independent of $e$. The continuity in $s=a$ and the jump condition in the derivative lead to the system of equations

$$
\begin{gathered}
A \cosh (\kappa a)+B_{e} \sinh (\kappa a)-C_{e} \cosh (\kappa(1-a))=0 \\
-\frac{1}{a} C_{e} \cosh \kappa(1-a)=\kappa\left(-A \sinh (\kappa a)-B_{e} \cosh (\kappa a)-C_{e} \sinh \kappa(1-a)\right) \\
-\frac{\beta}{a^{2}} A=\kappa \sum_{e} B_{e} .
\end{gathered}
$$

With the permutational invariance in mind, let us first analyse the situation with symmetric coefficients, $A, B=B_{e}, C=C_{e}$. Then $\sum_{e} B_{e}=n B$ and the corresponding coefficient matrix for $A, B$ and $C$ vanishes iff

$$
\frac{\beta}{a^{2}}(\sinh (\kappa a) \cosh \kappa(1-a)-a \kappa \cosh \kappa)+n \kappa(\kappa a \sinh \kappa-\cosh (\kappa a) \cosh \kappa(1-a))=0
$$


leading to an eigenvalue $\lambda=-\kappa(a)^{2}$ of multiplicity one. It can be seen that $\kappa(a)$ is bounded, and that the above equation reduces to (4.2) as $a \rightarrow \infty$. The other eigenvalues can be obtained from $B$ and $C$ as follows: set $\Theta_{n}:=\mathrm{e}^{2 \pi \mathrm{i} / n}$. Then for $k=1, \ldots, n-1$, we have the coefficients $B_{e, k}=\Theta_{n}^{e \cdot k} B$ and $C_{e, k}=\Theta_{n}^{e \cdot k} C, e=1, \ldots, n$. Since

$$
\sum_{e=1}^{n} B_{e, k}=B \sum_{e=0}^{n-1} \Theta_{n}^{e \cdot k}=0
$$

for $k=1, \ldots, n-1$, we finally arrive at a coefficient matrix similar to the previous one, but with $n$ replaced by zero. Consequently, if there were additional negative eigenvalues $\lambda=-\kappa(a)^{2}$, they would be of multiplicity $n-1$ and given by the relation

$$
\sinh (\kappa a) \cosh \kappa(1-a)-\kappa a \cosh \kappa=0 .
$$

But this equation has no solutions for $0<a \leq 1$ and $\kappa>0$. We skip the proof of this fact here.

For the second part, assume that $\beta \geq 0$. It is sufficient to calculate the Rayleigh quotient for the constant test function $f=\mathbb{1} \in \mathrm{H}^{1}(G)$ which yields

$$
\frac{\mathfrak{h}^{\beta, a}(f)}{\|f\|^{2}}=-\frac{1}{n}\left(\frac{\beta}{a^{2}}+\frac{1}{a}\right)
$$

being of order $\mathrm{O}\left(a^{-2}\right)$ if $\beta<0$ and of order $\mathrm{O}\left(a^{-1}\right)$ if $\beta=0$, negative in both cases; recall that $n=\operatorname{deg} v$.

Similarly, we expect the same behaviour for the operators on the manifold.

Lemma 4.5. If $\beta \geq 0$, then the spectrum of $H_{\varepsilon}^{\beta}$ is asymptotically unbounded from below, i.e.,

$$
\inf \sigma\left(H_{\varepsilon}^{\beta}\right) \rightarrow-\infty \quad \text { as } \quad \varepsilon \rightarrow 0
$$

Proof. Again, we plug the constant test function $u=\mathbb{1}$ into the Rayleigh quotient and obtain

$$
\frac{\mathfrak{h}_{\varepsilon}^{\beta}(u)}{\|u\|^{2}}=-\frac{\beta \varepsilon^{-2 a}+\varepsilon^{-a}}{n\left(1+\varepsilon+\varepsilon^{\alpha}\right)+\varepsilon \operatorname{vol} X_{v_{0}}}
$$

which obviously tends to $-\infty$ as $\varepsilon \rightarrow 0$.

Remark 4.6. As for a counterpart to the other claim in Lemma 4.4, the proof of the uniform boundedness from below as $\varepsilon \rightarrow 0$ for $\beta<0$ seems to need quite subtle estimates to compare the effect of the two competing potentials on $X_{\varepsilon, v_{0}}$ and $X_{\varepsilon, v_{e}}$ having strength proportional to $|\beta| \varepsilon^{-2 \alpha}$ and $\varepsilon^{-\alpha}$, respectively. Since the positive contribution $Q_{\varepsilon, v_{0}}=$ $|\beta| \varepsilon^{-1-2 \alpha}$ is more singular than the negative contributions $Q_{\varepsilon, v_{e}}=-\varepsilon^{-1-\alpha}$, we expect that the threshold of the spectrum remains bounded as $\varepsilon \rightarrow 0$.

We can now prove our second main result. For the $\delta_{\mathrm{s}}^{\prime}$-coupling Hamiltonian $H_{\beta}$ and the approximating operator $H_{\varepsilon}^{\beta}$ defined in (4.1) and (4.3), respectively, we make the following claim.

Theorem 4.7. Assume that $0<\alpha<1 / 13$, then

$$
\left\|\left(H_{\varepsilon}^{\beta}-\mathrm{i}\right)^{-1} J-J\left(H^{\beta}-\mathrm{i}\right)^{-1}\right\| \rightarrow 0
$$

as $\varepsilon \rightarrow 0$.

Proof. Denote by $H^{\beta, \varepsilon}=H^{\beta, a_{\varepsilon}}$ the $\varepsilon$-depending intermediate Hamiltonian on the metric graph with $\delta$-potentials of strength depending on $\varepsilon$ as defined before. For the corresponding graph and manifold model, the lower bound to lengths depends now on $\varepsilon$, 
specifically, $\ell_{-}=a_{\varepsilon}=\varepsilon^{\alpha}$. Moreover, from the definition of the constants $C_{1 / 2} \leq \widetilde{C}_{1 / 2}$ and $\varepsilon_{1 / 2}$ in (2.15) and from Lemma 3.2, we conclude that

$\widetilde{C}_{1 / 2}=\widetilde{C}_{1 / 2}(\varepsilon)=\mathrm{O}\left(\varepsilon^{-4 \alpha}\right), \quad \varepsilon_{1 / 2}=\varepsilon_{1 / 2}(\varepsilon)=\mathrm{O}\left(\varepsilon^{3 \alpha}\right) \quad$ and $\quad \delta=\delta_{\varepsilon}=\mathrm{O}\left(\varepsilon^{(1-5 \alpha) / 2}\right)$.

Note that the dominant term in the closeness-error $\delta_{\varepsilon}$ (see (3.9) ) is the last one containing the potential. From Theorem 3.3 it follows now that

$$
\left\|\left(H_{\varepsilon}^{\beta}-\mathrm{i}\right)^{-1} J-J\left(H^{\beta, \varepsilon}-\mathrm{i}\right)^{-1}\right\| \leq 10 \delta_{\varepsilon} \max \left\{\widetilde{C}_{1 / 2}(\varepsilon), \sqrt{2}\right\}=\mathrm{O}\left(\varepsilon^{(1-13 \alpha) / 2}\right) .
$$

so that Theorem 4.3 yields the sought conclusion. Note that the exponent of $\varepsilon$ in $\delta_{\varepsilon} \widetilde{C}_{1 / 2}(\varepsilon)$ is $(1-5 \alpha) / 2-4 \alpha=(1-13 \alpha) / 2>0$ provided $0<\alpha<1 / 13$.

We can now proceed and state similar results as in Theorems 3.4 3.7 for the $\delta_{\mathrm{s}}^{\prime}$ approximation by using arguments similar to those in [P06, App.] or [P08], where only non-negative operators were considered (covering, as usual, operators bounded uniformly from below by a suitable shift). In our present situation, we can only guarantee the resolvent convergence at non-real points like $z=\mathrm{i}$. Nevertheless, the arguments in [P06, App.] or [P08] can be used to conclude the convergence of suitable functions of operators as well as the convergence of the dimension of spectral projections.

Remark 4.8. Note that the asymptotic lower unboundedness of $H_{\varepsilon}^{\beta}$ (and of the intermediate operator $H^{\beta, \varepsilon}$ ) for $\beta \geq 0$ described in Lemmata 4.4 and 4.5 is not a contradiction to the fact that the limit operator $H^{\beta}$ is non-negative. For example, the spectral convergence of Theorem 3.5 holds only for compact intervals $I \subset \mathbb{R}$. In particular, $\sigma\left(H^{\beta}\right) \cap I=\emptyset$ implies that

$$
\sigma\left(H_{\varepsilon}^{\beta}\right) \cap I=\emptyset \quad \text { and } \quad \sigma\left(H^{\beta, \varepsilon}\right) \cap I=\emptyset
$$

provided $\varepsilon>0$ is sufficiently small. This spectral convergence means that the negative spectral branches of $H_{\varepsilon}^{\beta}$ all have to tend to $-\infty$.

Acknowledgement. O.P. enjoyed the hospitality in the Doppler Institute where a part of the work was done. The research was supported by the Czech Ministry of Education, Youth and Sports within the project LC06002.

\section{REFERENCES}

[ACF07] S. Albeverio, C. Cacciapuoti, and D. Finco, Coupling in the singular limit of thin quantum waveguides, J. Math. Phys. 48 (2007), 032103.

[CE07] C. Cacciapuoti and P. Exner, Nontrivial edge coupling from a Dirichlet network squeezing: the case of a bent waveguide, J. Phys. A 40 (2007), L511-L523.

[CE04] T. Cheon and P. Exner, An approximation to $\delta^{\prime}$ couplings on graphs, J. Phys. A 37 (2004), no. 29, L329-L335.

[CS98] T. Cheon and T. Shigehara, Realizing discontinuous wave functions with renormalized shortrange potentials, Phys. Lett. A 243 (1998), 111-116.

[DEK01] P. Duclos, P. Exner, and D. Krejčiřík, Bound states in curved quantum layers, Comm. Math. Phys. 223 (2001), no. 1, 13-28.

[DT06] G. F. Dell'Antonio and L. Tenuta, Quantum graphs as holonomic constraints, J. Math. Phys. 47 (2006), no. 7, 072102, 21.

[E97] P. Exner, A duality between Schrödinger operators on graphs and certain Jacobi matrices, Ann. Inst. H. Poincaré Phys. Théor. 66 (1997), no. 4, 359-371.

[EKK $\left.{ }^{+} 08\right]$ P. Exner, J. P. Keating, P. Kuchment, T. Sunada, and A. Teplayaev (eds.), Analysis on graphs and its applications, Proc. Symp. Pure Math., vol. 77, Providence, R.I., Amer. Math. Soc., 2008.

[ENZ01] P. Exner, H. Neidhardt, and V.A. Zagrebnov, Potential approximations to $\delta^{\prime}$ : an inverse Klauder phenomenon with norm-resolvent convergence, Comm. Math. Phys. 224 (2001), no. 3, 593-612.

[EP05] P. Exner and O. Post, Convergence of spectra of graph-like thin manifolds, Journal of Geometry and Physics 54 (2005), 77-115. 
[EP07] _ Convergence of resonances on thin branched quantum wave guides, J. Math. Phys. 48 (2007), 092104+43.

[EP08] Q Quantum networks modelled by graphs, Quantum Few-Body Systems, AIP Conf. Proc., vol. 998, Amer. Inst. Phys., Melville, NY, 2008, pp. 1-17.

[ET06] P. Exner and O. Turek, Approximations of permutation-symmetric vertex couplings in quantum graphs, Quantum graphs and their applications, Contemp. Math., vol. 415, Amer. Math. Soc., Providence, RI, 2006, pp. 109-120.

[ET07] - Approximations of singular vertex couplings in quantum graphs, Rev. Math. Phys. 19 (2007), no. 6, 571-606.

[FW93] M. I. Freidlin and A. D. Wentzell, Diffusion processes on graphs and the averaging principle, Ann. Probab. 21 (1993), no. 4, 2215-2245.

[G08] D. Grieser, Spectra of graph neighborhoods and scattering, Proc. London Math. Soc. (3) 97 (2008), no. 3, 718-752.

[HN99] I. Herbst and S. Nakamura, Schrödinger operators with strong magnetic fields: Quasiperiodicity of spectral orbits and topology, American Mathematical Society. Transl., Ser. 2, Am. Math. Soc. 189(41) (1999), 105-123.

[Ku04] P. Kuchment, Quantum graphs: I. Some basic structures, Waves Random Media 14 (2004), S107-S128.

[KuZ01] P. Kuchment and H. Zeng, Convergence of spectra of mesoscopic systems collapsing onto a graph, J. Math. Anal. Appl. 258 (2001), no. 2, 671-700.

[MV07] S. Molchanov and B. Vainberg, Scattering solutions in networks of thin fibers: small diameter asymptotics, Comm. Math. Phys. 273 (2007), no. 2, 533-559.

[Pa07a] B. Pavlov, Neumann Schrödinger 2D junction: collapsing on a quantum graph: a generalized Kirchhoff boundary condition, Preprint (2007).

[Pa07b] _ _ A star-graph model via operator extension, Math. Proc. Cambridge Philos. Soc. 142 (2007), 365-384.

[P05] O. Post, Branched quantum wave guides with Dirichlet boundary conditions: the decoupling case, Journal of Physics A: Mathematical and General 38 (2005), no. 22, 4917-4931.

[P06] _ Spectral convergence of quasi-one-dimensional spaces, Ann. Henri Poincaré 7 (2006), no. 5, 933-973.

[P08] S S S S _ Sectral analysis on graph-like spaces, Habilitation thesis in preparation (2008).

[RS01] J. Rubinstein and M. Schatzman, Variational problems on multiply connected thin strips. I. Basic estimates and convergence of the Laplacian spectrum, Arch. Ration. Mech. Anal. 160 (2001), no. 4, 271-308.

[Sai00] Y Saito, The limiting equation for Neumann Laplacians on shrinking domains., Electron. J. Differ. Equ. 31 (2000), 25 p.

Department of Theoretical Physics, NPI, Academy of Sciences, 25068 Řě̌ near Prague, and Doppler Institute, Czech Technical University, Břehová 7, 11519 Prague, Czechia

E-mail address: exner@ujf.cas.cz

Institut für Mathematik, Humboldt-Universität Zu Berlin, Rudower Chaussee 25, 12489 Berlin, Germany

E-mail address: post@math.hu-berlin.de 